
населения

УДК 303.725.34(083.41)

DOI: $10.18101 / 2304-4446-2019-4-37-42$

\title{
ПОДХОДЫ К МОДЕЛИРОВАНИЮ МУЛЬТИПЛИКАТОРОВ ДОХОДОВ И РАСХОДОВ НАСЕЛЕНИЯ
}

\section{(C) Дугаржапова Долгорма Баторовна}

кандидат экономических наук, научный сотрудник, Бурятский научный центр Сибирского отделения РАН; Россия, 670047, г. Улан-Удэ, ул. Сахьяновой, 8

доцент,

Бурятский государственный университет имени Доржи Банзарова

Россия, 670000, г. Улан-Удэ, ул. Смолина, 24а

E-mail: dolgor@mail.ru

В статье рассмотрены подходы к получению количественных оценок мультипликативных эффектов в современной экономике. Рассмотрены возможности использования модели Миядзавы, включающей в состав эндогенных параметров потребление домашних хозяйств, сгруппированных по источникам получения доходов для оценки экономических мультипликативных эффектов в экономике. В общем виде представлена методика перехода матрицы в систему мультипликаторов, позволяющих проводить анализ взаимосвязей воспроизводственной системы и институциональных секторов, а также прогнозировать влияние экзогенных факторов на показатели динамики роста доходов и потребительских расходов отдельных квинтильных групп населения. Даны сценарные расчеты мультипликативного изменения основных макроэкономических показателей экономики Республики Бурятия при приросте автономного конечного спроса на продукцию в разрезе 15 видов экономической деятельности.

Ключевые слова: балансовая модель; модель Миядзавы; эндогенные параметры; доходы; потребительские расходы; домохозяйства; мультипликатор; виды экономической деятельности; региональная экономика.

\section{Для цитирования}

Дугаржапова Д. Б. Подходы к моделированию мультипликаторов доходов и расходов населения // Вестник Бурятского государственного университета. Экономика и менеджмент. 2019. № 4. С. 37-42.

Принятие эффективных и целесообразных управленческих решений на региональном уровне имеет огромное значение в условиях современного развития экономики. Для эффективного регулирования необходимо учитывать особенности региональной структуры производства, формирования и распределения доходов и потребления всех секторов экономики региона. Кроме того, оценка развития экономики региона и ее отдельных секторов невозможна без учета воздействия одних параметров экономической системы на изменения других экономических показателей [2]. В этой связи инструменты комплексного анализа и прогнозирования экономической системы, способные отразить взаимодействие всех ее подсистем и отдельных элементов, имеют особое значение.

Среди отечественных и зарубежных исследователей используются различные подходы по определению причинно-следственных связей в экономике, включая мультипликатор Дж. Кейнса и его последователей [3]. Мультипликационные эф- 
фекты представлены в агрегированном виде, что обусловлено их расчетом в форме скаляра.

Альтернативным подходом являются матричные мультипликаторы, в том числе межотраслевой баланс (таблицы «затраты-выпуск»), разработанный В. Леонтьевым [4]. В классическом виде межотраслевой баланс демонстрирует взаимосвязь между вектором конечного спроса и валовыми выпусками отраслей.

Вместе с тем матрица полных затрат, построенная по модели Леонтьева, является мультипликатором валового продукта, а не мультипликатором дохода. В то время как ввод показателей потребления домашних хозяйств в состав эндогенных параметров приводит к увеличению масштабов действия механизма мультипликации [5; 6]. Таким образом, одним из путей расширения модели МОБ является эндогенное задание вектора конечного спроса, в частности, через модель потребительского поведения населения [1].

Одним из подходов по эндогенизации домашних хозяйств в модели «затраты - выпуск» является межотраслевая расширенная модель Миядзавы $[7,9,10]$.

Матричная модель Миядзавы имеет вид:

$$
\left(\begin{array}{l}
x \\
Y
\end{array}\right)=\left(\begin{array}{ll}
A & C \\
V & 0
\end{array}\right)\left(\begin{array}{l}
x \\
Y
\end{array}\right)+\left(\begin{array}{l}
f \\
g
\end{array}\right)
$$

Здесь А - это матрица коэффициентов прямых затрат; $\mathrm{x}$ - валовой выпуск, $\mathrm{f}$ - конечный спрос, вектор Y представляет собой общий доход, матрица V соотношение домашних хозяйств на добавленную стоимость; вектор g - экзогенный доход; матрица $\mathrm{C}$ - расходы на потребление.

Если допустить, что $\underset{(q \times 1)}{g}=0$, тогда система уравнений (1) примет вид:

$$
\begin{aligned}
& x=A x+C y+f^{*}, \text { при } y=V x, \\
& {\left[\begin{array}{l}
x \\
y
\end{array}\right]=\left[\begin{array}{cc}
I-A & -C \\
-V & I
\end{array}\right]^{-1}\left[\begin{array}{c}
f^{*} \\
0
\end{array}\right] .}
\end{aligned}
$$

Элементы системы (3) могут быть разложены следующим образом:

$$
\left[\begin{array}{l}
x \\
y
\end{array}\right]=\left[\begin{array}{cc}
B\left[I+C(I-V B C)^{-1} V B\right] & B C(I-V B C)^{-1} \\
(I-V B C)^{-1} V B & (I-V B C)^{-1}
\end{array}\right]\left[\begin{array}{c}
f^{*} \\
0
\end{array}\right],
$$

где $B=(I-A)^{-1}$ - классическая обратная межотраслевая матрица Леонтьева.

Данная система может быть упрощена. Если в уравнение (4) подставить $V B C=L$ и $K=(I-L)^{-1}=(I-V B C)^{-1}$, тогда уравнение (4) примет вид:

$$
\left[\begin{array}{l}
x \\
y
\end{array}\right]=\left[\begin{array}{cc}
B\left[\begin{array}{c}
I+C K V B] \\
(n \times n)
\end{array}\right. & \underset{(n \times q)}{B C K} \\
\underset{(q \times n)}{K V B} & \underset{(q \times q)}{K}
\end{array}\right]\left[\begin{array}{c}
f^{*} \\
0
\end{array}\right] .
$$

$\mathrm{L}=\mathrm{VBC}$ - является междоходной матрицей коэффициентов, а также

$$
\mathrm{K}=(1-\mathrm{L})^{-1}=(1-\mathrm{VBC})^{-1}=1+\mathrm{V} \Delta \mathrm{C}
$$

К - обобщенный мультипликатор Кейнса, и

$$
\Delta=(1-\mathrm{A}-\mathrm{CV})^{-1}=\mathrm{B}+\mathrm{BCKVB}
$$

представляет собой увеличенную обратную Леонтьева. 

населения

KVB - матрица межотраслевых мультипликаторов дохода, отражающая прямой, косвенный и индуцированный доходы для каждой доходной группы, созданные в результате удовлетворения конечного спроса в экономике.

Особенностью матричной модели Миядзавы является построение многосекторного мультипликатора, сформированного путем включения процесса генерации дохода [8].

Тем не менее это расширение стандартной модели Леонтьева может неадекватно учитывать взаимосвязь между межотраслевой и потребительской структурами. Причина заключается в том, что структура потребления в целом зависит от структуры распределения доходов, которая регулирует структуру потребления в том смысле, что структура потребления состоит из поведения расходов различных групп доходов.

Информационной базой формирования матричной модели Миядзавы являются региональные таблицы «затраты-выпуск» за 2011 г. по Республике Бурятия в разрезе 15 видов экономической деятельности.

С помощью статистических данных о заработной плате, начисленной работникам по видам экономической деятельности в пределах 21 интервального диапазона, и потребительских расходов домохозяйств в разрезе 20-процентных групп населения с различным уровнем располагаемых ресурсов по Республике Бурятия за 2011 г. произведен расчет матрицы удельного потребления благ группами домашних хозяйств в расчете на $1 \mathrm{p}$. доходов по каждой группе $C_{(15 \times 5)}$ и матрицы удельных доходов каждой группы домашних хозяйств в расчете на 1 p. стоимости валовых выпусков по видам экономической деятельности $V_{(5 \times 15)}$. Расчеты расходов на конечное потребление на основе агрегированных данных о потребительских расходах домохозяйств по 20-процентным группам населения с различным уровнем располагаемых ресурсов в разрезе выделенных видов экономической деятельности сбалансированы с данными расходов на конечное потребление домохозяйств таблиц «затраты — выпуск» по Республике Бурятия за 2011 г.

На основе полученных матриц $C$ и $V$ и матрицы коэффициентов прямых затрат $A$ была построена расширенная матрица коэффициентов $\bar{A}=\left[\begin{array}{cc}\underset{(n \times n)}{A} & \underset{(n \times q)}{C} \\ \underset{(q \times n)}{V} & 0 \\ (q \times q)\end{array}\right]$. Применив к ней стандартное обращение Леонтьева $(I-A)^{-1}$, был получен набор мультипликаторов K и L (табл.1).

Матрицы прямых $L$ и полных $K$ мультипликаторов дохода

\begin{tabular}{|c|c|c|c|c|c|c|c|c|c|c|}
\hline \multirow{2}{*}{$\begin{array}{c}\text { Доходные } \\
\text { группы }\end{array}$} & \multicolumn{9}{|c|}{ Матрица $L$} & \multicolumn{5}{c|}{ Матрица $K$} \\
\hline 1-я & 0.011 & 0.011 & 0.015 & 0.012 & 0.015 & 1.017 & 0.017 & 0.023 & 0.018 & 0.023 \\
\hline 2-я & 0.020 & 0.020 & 0.027 & 0.022 & 0.028 & $\mathbf{0 . 0 3 1}$ & 1.032 & 0.041 & 0.033 & 0.042 \\
\hline 3-я & 0.030 & 0.030 & 0.038 & 0.030 & 0.038 & 0.046 & 0.046 & 1.058 & 0.046 & 0.058 \\
\hline 4-я & 0.069 & 0.070 & 0.086 & 0.068 & 0.088 & 0.106 & 0.107 & 0.132 & 1.105 & 0.133 \\
\hline 5-я & 0.171 & 0.169 & 0.204 & 0.161 & 0.200 & 0.257 & 0.255 & 0.310 & 0.245 & 1.305 \\
\hline
\end{tabular}

Произведенные расчеты показывают более высокий эффект капитализации доходов у групп населения с наибольшим уровнем дохода по сравнению с груп- 
пами населения с более низким доходом в результате увеличения конечного спроса на продукцию отраслей экономики региона. Так, например, прямой рост доходов в 1-й группе домохозяйств на 1 р. приводит к увеличению доходов домохозяйств 2-й группы на 0,031 p.

Сценарные расчеты мультипликативного изменения основных макроэкономических показателей экономики Республики Бурятия при приросте (на 100 тыс. руб.) автономного конечного спроса на продукцию (услугу) каждого из видов экономической деятельности представлены в таблице 2.

Таблица 2

Мультипликативный прирост основных макроэкономических показателей при увеличении на 100 тыс. р. конечного автономного спроса на продукцию

(услугу) по видам экономической деятельности ${ }^{1}: \Delta f_{i}(i=1, \ldots, 15)$, тыс. p.

\begin{tabular}{|c|c|c|c|c|c|}
\hline $\begin{array}{c}\text { Коды } \\
\text { Вэд }\end{array}$ & $\begin{array}{c}\text { Промежуточное } \\
\text { потребление }\end{array}$ & $\begin{array}{c}\text { Потребление } \\
\text { домашних } \\
\text { хозяйств }\end{array}$ & $\begin{array}{c}\text { Доходы } \\
\text { домашних } \\
\text { хозяйств }\end{array}$ & $\begin{array}{c}\text { Конечное } \\
\text { потребление }\end{array}$ & $\begin{array}{c}\text { Совокупный } \\
\text { валовый выпуск }\end{array}$ \\
\hline A-1 & 126,76 & 37,83 & 34,98 & 137,83 & 264,59 \\
\hline B-2 & 166,72 & 55,92 & 51,80 & 155,92 & 322,64 \\
\hline C-3 & 299,74 & 159,61 & 145,42 & 259,61 & 559,35 \\
\hline D-4 & 180,50 & 43,81 & 40,99 & 143,81 & 324,31 \\
\hline E-5 & 339,83 & 140,18 & 128,46 & 240,18 & 580,01 \\
\hline F-6 & 178,16 & 41,89 & 38,60 & 141,89 & 320,05 \\
\hline G-7 & 125,60 & 31,87 & 29,51 & 131,87 & 257,47 \\
\hline H-8 & 155,48 & 44,63 & 41,20 & 144,63 & 300,10 \\
\hline I-9 & 177,40 & 67,81 & 62,49 & 167,81 & 345,21 \\
\hline J-10 & 382,48 & 265,76 & 246,32 & 365,76 & 748,24 \\
\hline K-11 & 173,03 & 74,35 & 68,75 & 174,35 & 347,39 \\
\hline L-12 & 178,34 & 86,68 & 81,01 & 186,68 & 365,02 \\
\hline M-13 & 199,30 & 100,54 & 94,94 & 200,54 & 399,84 \\
\hline N-14 & 159,27 & 68,00 & 64,57 & 168,00 & 327,27 \\
\hline O-15 & 179,71 & 101,46 & 95,39 & 201,46 & 381,17 \\
\hline
\end{tabular}

${ }^{1}$ Виды экономической деятельности:

A-1 Сельское хозяйство, охота и лесное хозяйство

В-2 Рыболовство, рыбоводство

C-3 Добыча полезных ископаемых (кроме газовой, угольной и минеральных ископаемых)

D-4 Обрабатывающие производства

E-5 Производство и распределение электроэнергии, газа и воды

F-6 Строительство

G-7 Оптовая и розничная торговля; ремонт автотранспортных средств, мотоциклов, бытовых изделий и предметов личного пользования

Н-8 Гостиницы и рестораны

I-9 Транспорт и связь

J-10 Финансовая деятельность

K-11 Операции с недвижимым имуществом, аренда и предоставление услуг (IT, наука, бухучет и аудит, архитектура, инженерное проектирование, геолого-разведочные работы и т.д.)

L-12 Государственное управление и обеспечение военной безопасности; обязательное социальное обеспечение

М-13 Образование

N-14 Здравоохранение и предоставление социальных услуг

0-15 Предоставление прочих коммунальных, социальных и персональных услуг 

населения

Согласно проведенным расчетам, увеличение на 100 тыс. p. автономного конечного спроса на продукцию сельского хозяйства в целом по Республике Бурятия приводит к увеличению промежуточного потребления на 126,76 тыс. р., конечного потребления - на 137,83 тыс. р., совокупного валового выпуска - на 264,59 тыс. p. При этом совокупные доходы всех домашних хозяйств возрастут на 34,98 тыс. p., а их совокупные расходы на потребление благ - на 37,83 тыс. p.

В сельском хозяйстве, так же как и в сфере оптовой и розничной торговли, изменения автономного спроса среди всех видов экономической деятельности оказывают наименьшее влияние на изменения макроэкономических показателей в Республике Бурятия. Наибольший прирост всех макроэкономических показателей обеспечивает увеличение автономного спроса на услуги финансовой деятельности (рост совокупного валового выпуска - на 748,24 тыс. р., конечного и промежуточного потребления - на 365,76 тыс. р. и 382,48 тыс. р. соответственно, доходов и потребления домашних хозяйств - на 246,32 тыс. р. и 265,76 тыс. p. соответственно). Также значительное влияние на прирост макроэкономических показателей оказывает увеличение автономного спроса в сфере производства и распределения электроэнергии и добычи полезных ископаемых.

Безусловно, результаты оценки мультипликаторов следует интерпретировать крайне осторожно для характеристики значимости тех или иных видов деятельности в экономике или для выбора отраслевых приоритетов при разработке экономической политики региона. Для этого требуется более глубокий анализ, который, помимо оценки мультипликативных эффектов прироста выпуска, должен включать рассмотрение других эффектов, в том числе и прироста капитальных затрат, сокращения безработицы, социальных трансфертов и т. д. [3].

Вместе с тем при разработке региональных программ развития необходимо учитывать особенности экономического поведения населения региона при изменении их финансового положения.

В этой связи предложенная методика оценки мультипликативных эффектов с учетом региональной структуры производства и доходов усиливает возможности использования балансовой модели, позволяя оценить воздействие эндогенных параметров на изменения макроэкономических показателей в разрезе отраслей региона.

\section{Литература}

1. Дондоков 3. Б.-Д., Дырхеев К. П. Межотраслевые модели с расширенным составом эндогенных переменных // Вестник Бурятского государственного университета. Вып. Экономика. Право. 2014. № 2. С. 3-5.

2. Ермакова С. Ю. Теория мультипликатора и мультипликационные эффекты в экономике: дис. ... канд. экон. наук. Улан-Удэ, 2006.

3. Оценка мультипликативных эффектов в российской экономике на основе таблиц «затраты-выпуск» / М. Ю. Ксенофонтов [и др.] // Проблемы прогнозирования. 2018. T. 167. № 2. C. 3-14.

4. Леонтьев В. Межотраслевая экономика. М.: Экономика, 1997. 477 с.

5. Михеева Н. Н. Матрицы социальных счетов: направления исследования и ограничения использования // ЭКО. 2011. № 6. С. 103-118.

6. Широв А. А., Янтовский А. А. Оценка мультипликативных эффектов в экономике: возможности и ограничения // ЭКО. 2011. № 2. С. 40-58. 
7. Expanded Miyazawa framework: Labor and Capital Income, Savings, Consumption, and Investment Links by Michael Sonis and Geoffrey J.D. Hewings. REAL 00-T-14 - December, 2000.

8. Geoffrey J. D. Hewings. Miyazawa's Contributions to Extended Input-Output Analysis: Introduction to the Special Sessions, 1999.

9. Miller R. E., Blair P. D. Input-Output Analysis: Foundations and Extensions. Cambridge University Press, 2009. 784 p.

10. Ronald E. Miller and Peter D. Blair. Input-Output Analyses. Foundation and Extensions. Second Edition. Cambridge University Press. The Edinburg Building, Cambridge CB2 8RU, UK, 2009.

\section{APPROACHES TO MODELING PERSONAL INCOME AND EXPENDITURE MULTIPLIERS}

Dolgorma B. Dugarzhapova

Cand. Sci. (Econ.), Researcher,

Buryat Scientific Center SB RAS

8 Sakhyanovoy St., Ulan-Ude 670047, Russia

A/Prof. of Accounting and Finance Department, Dorzhi Banzarov Buryat State University

24a Smolina St., Ulan-Ude 670000, Russia

E-mail: dolgor@mail.ru

The article presents the approaches to quantitative assessment of multiplier effects in modern economy. We have considered the possibilities of using the Miyazawa model, which includes consumption of households grouped by income sources in endogenous parameters, for assessing multiplier effects in the economy. The article presents in general terms the method for transition matrix into a system of multipliers, which allows analyzing the correlation between the reproductive system and institutional sectors, as well as predicting the influence of exogenous factors on the growth of income and consumer spending dynamics of individual income quintile groups. We have proposed scenario calculations of multiplier changes in the main macroeconomic indicators of Buryatia's economy in increased autonomous final demand for products broken down by 15 economic sectors.

Keywords: balance model; the Miyazawa model; endogenous parameters; income; consumer expenditure; households; multiplier; economic sectors; regional economy. 